\title{
Improvements to Wire Bundle Thermal Modeling for Ampacity Determination
}

\author{
Steven L. Rickman ${ }^{1}$, Christopher J. Iannello ${ }^{2}$, Khadijah Shariff ${ }^{3}$ \\ ${ }^{1}$ NASA Engineering and Safety Center \\ 2101 NASA Parkway, Houston, Texas, USA \\ steven.1.rickman@nasa.gov; christopher.j.iannello@nasa.gov \\ ${ }^{2}$ NASA Engineering and Safety Center \\ Kennedy Space Center, SR 405, Titusville, Florida, USA \\ ${ }^{3}$ NASA Johnson Space Center \\ 2101 NASA Parkway, Houston, Texas, USA \\ khadijah.i.shariff@nasa.gov
}

\begin{abstract}
Determining current carrying capacity (ampacity) of wire bundles in aerospace vehicles is critical not only to safety but also to efficient design. Published standards provide guidance on determining wire bundle ampacity but offer little flexibility for configurations where wire bundles of mixed gauges and currents are employed with various external insulation jacket surface properties. Thermal modeling has been employed in an attempt to develop techniques to assist in ampacity determination for these complex configurations. An earlier tool allowed analysis of wire bundle configurations but was constrained to configurations comprised of less than 50 elements. Additionally, for vacuum analyses, configurations with very low emittance external jackets suffered from numerical instability in the solution. A new thermal modeler is presented allowing for larger configurations and is not constrained by low bundle jacket surface infrared emittance calculations. Formulation of key internal radiation and interface conductance parameters is discussed including the effects of temperature and ambient air pressure on wire-to-wire thermal conductance. Test cases comparing model-predicted ampacity and that calculated from standards documents are presented.
\end{abstract}

Keywords: Ampacity, wire bundle, heat transfer, thermal analysis

\section{Introduction}

Ampacity, a term for amperage capacity, is a measure of the current carrying capability of a wire or a collection of wires in a bundle. Current practice relies on the use of published standards (e.g., [1]) to derate, both, single wires and bundles. However, use of the standards is limited as none of the publicly-available standards provide a procedure to assess the effect of a smart short within a bundle, nor do they allow for mixed wire sizes, different wire jacket emittances, or a variety of currents on individual conductors. Pursuit of an analytical approach is highly desirable to allow assessment of real world configurations not readily addressed by the standards.

For a single wire, steady state heat transfer and the resulting conductor temperature is readily calculated by establishing a heat balance, i.e., the rate at which is heat generated within the wire due to ohmic heating must be equal to the rate at which it is rejected from the wire. For a sufficiently long wire, heat losses through the end terminations may be neglected and the heat leaving the wire is by convection and radiation from the wire insulation jacket. For wire bundles, characterization of the heat transfer is complicated due to the presence of multiple heat generating wires and interface conductances, radiation and even gas conduction between adjacent wires. Various studies have been performed by [2] and [3]. The complexities introduced with the bundle configuration result in a more tortuous heat transfer path from wires deep within the bundle to the free surface where heat is rejected from the bundle. Heat transfer considerations for wire-to-wire heat transfer within a bundle are presented. The capability to model wire bundles has been extended from the methodology presented in [4] in the form of a complex wire bundle thermal model builder. In this work, the model builder has been used to formulate three analytical configurations for use in comparison with a published derating standard. 


\section{Thermal Modeling}

\subsection{Wire to Wire Heat Transfer within Wire Bundles}

Reference [4] provided the derivation of the thermal network representing heat transfer within a wire bundle and noted wire-to-wire heat transfer between adjacent wire insulation jackets consisted of, both, radiative and conductive heat transfer paths. Heat transfer between adjacent wires can occur due to direct contact between adjacent insulation jackets, via radiation and also via air conduction in the gaps between wires for cases where an atmosphere is present. A typical thermal network between wire jackets for two adjacent wires is shown in Figure 1. While the radiation conductance between adjacent wire jackets can be expressed as a fixed value, bench testing has suggested that the overall interface conductance $\left(G_{\text {int }}\right)$, is a function of, both, temperature and pressure. Thermal radiation conductance $\left(G_{\text {rad }}\right)$, contact conductance $\left(G_{\text {contact }}\right)$ and air conductance $\left(G_{\text {air }}\right)$ are discussed in the following sections.

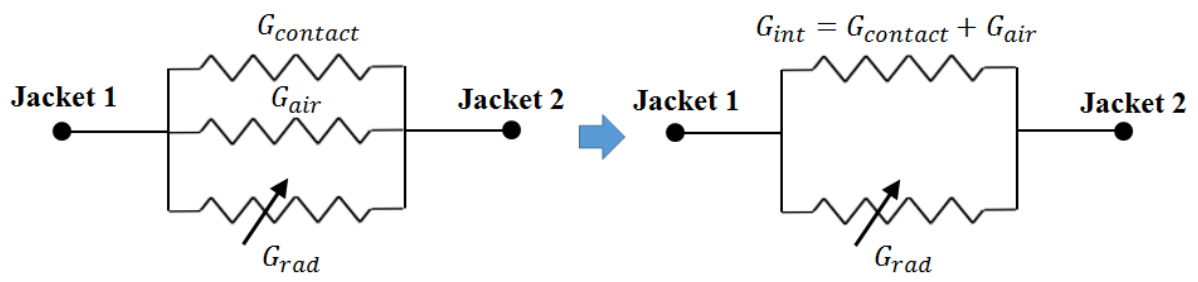

Fig. 1: Typical heat transfer network between adjacent wire jackets.

\subsubsection{Radiation Heat Transfer Between Adjacent Wire Jackets}

The general form of radiation heat transfer between two objects is given by:

$$
\dot{Q}_{r a d, 1-2}=G_{r a d}\left(T_{1}^{4}-T_{2}^{4}\right)
$$

where the radiation conductance, $G_{r a d}=\varepsilon_{1} B_{12} \sigma A_{1}, \varepsilon_{1}$ is the emittance of wire $1, B_{12}$ is the radiation interchange factor between wire jackets 1 and 2, $\sigma$ is the Stefan-Boltzmann constant and $A_{1}$ is the surface area of wire jacket 1 .

Implementation of wire jacket-to-wire jacket radiation within the thermal model assumed the following:

a. Only first order radiation heat transfer was considered (i.e., direct radiation heat transfer between adjacent wire jackets with no reflection off of other wire jackets in view of the two wires of interest);

b.Both wire jackets are assumed to have the same infrared emittance;

c. Diffuse radiation heat transfer.

Under these assumptions, $G_{\text {rad }}$ values were calculated for the range of possible wire size combinations (using the Cullimore and Ring Technologies $\operatorname{RadCAD}^{\circledR}$ application) by modeling adjacent wire jackets as infinite cylinders and normalizing the results based on radius ratios (Figure 2).

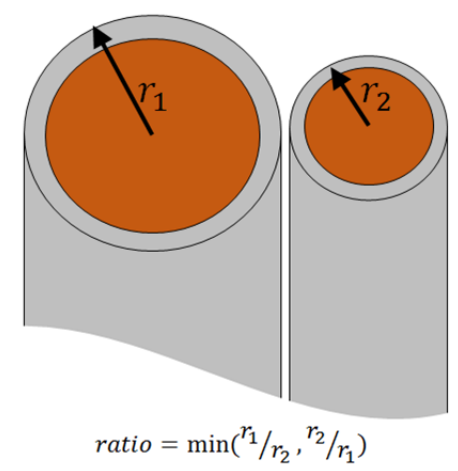

Fig. 2: Analysis configuration and radius ratio. 
Results were formatted into a spreadsheet look-up table across the range of expected radius ratios and emittances. When a wire bundle analysis configuration is changed, updating of the $B_{i j}$ matrix (i.e., the interchange factor between wires $i$ and $j$ ) is required. For each pair of wires, the radius ratio is calculated and then a look-up of the corresponding value is performed based on the wire jacket emittance. When the radius ratio exceeds that for which $B_{i j}$ data is calculated, reciprocity is used, namely:

$$
\varepsilon_{i} A_{i} B_{i j}=\varepsilon_{j} A_{j} B_{j i}
$$

where, again, $\varepsilon$ and $A$ are the emittances and wire jacket surface areas, respectively, for wires $i$ or $j$. The radiation conductance between two adjacent wire jackets, $\left(G_{r a d}\right)_{i j}$ is:

$$
\left(G_{r a d}\right)_{i j}=\sigma \varepsilon_{i} A_{i} B_{i j}
$$

\subsubsection{Variation of Contact Conductance with Wire Bundle Temperature}

The remaining heat transfer paths are linear in nature (i.e., heat transfer from one jacket to another is linear function of $\Delta T)$. The heat transfer paths comprising $G_{i n t}$, specifically, $G_{\text {contact }}$ and $G_{\text {air }}$ may be considered to be in parallel with one another. Between two insulation jackets the aggregate interface conductance is given by:

$$
G_{\text {int }}=G_{\text {contact }}+G_{\text {air }}
$$

Heat transfer paths between three adjacent wire jackets are depicted in Figure 3.
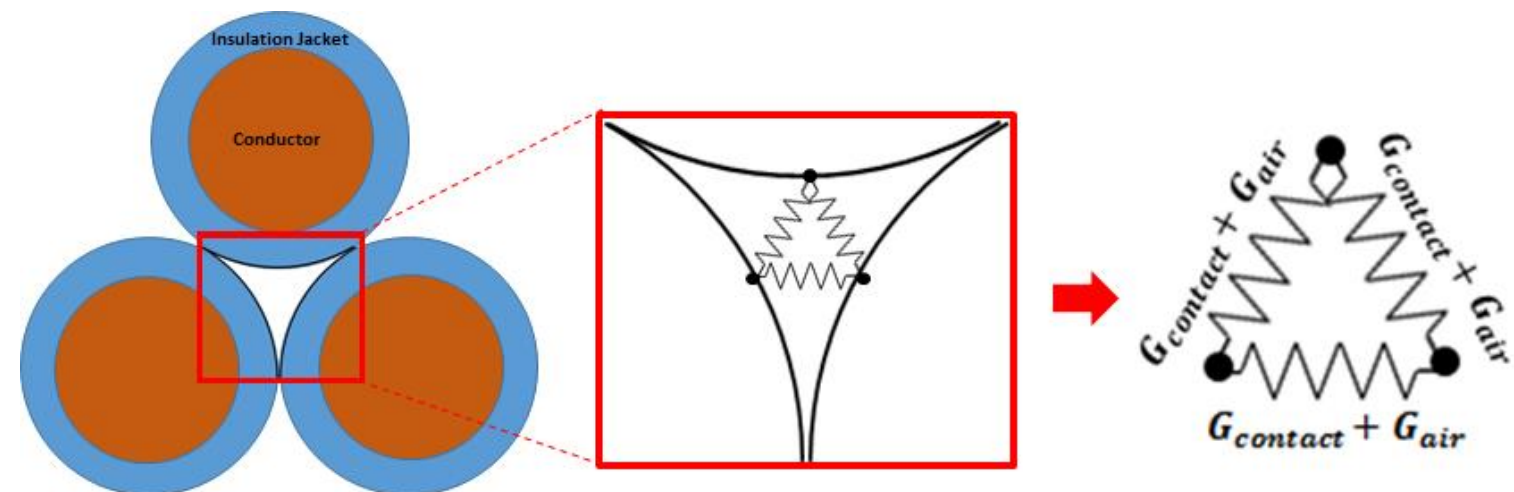

Fig. 3: Schematic depicting contact conductance and air conductance between adjacent wires.

Bench testing of wire bundles over a range of background currents for, both, in-air and vacuum conditions provided opportunities to correlate the thermal model for a large bundle comprised of approximately one hundred wires with a mixture of ethylene tetrafluoroethylene (ETFE) jacketed, 20 American Wire Gauge (AWG) and 22 AWG sizes. By varying the wire background current a correlation between the wire bundle bulk average temperature and interface conductance was found by changing the interface conductance in the thermal model based on the calculated bundle bulk average temperature by trial and error. A curve fit to the interface conductance data for the in-air analysis cases was performed and showed excellent correlation with a parabola $\left(R^{2}=0.99834\right)$, the shape of which is depicted in Figure 4 . 


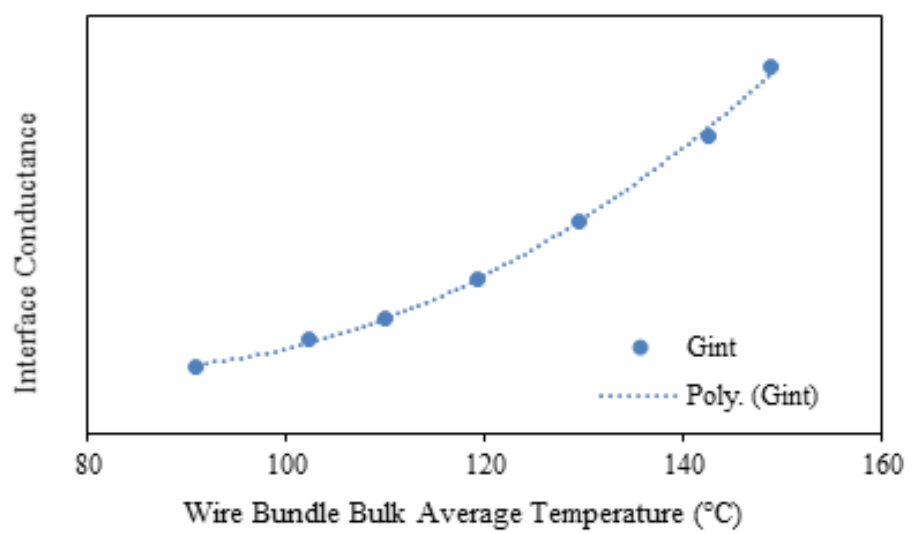

Fig. 4: Curve depicting the shape of wire jacket-to-wire jacket interface conductance versus wire bundle bulk average temperature.

Once established, the equation was used to generate $G_{i n t}$ for bundle bulk average temperatures up to $200^{\circ} \mathrm{C}$. These data became the interface conductance values used in the thermal model over the use range of $20^{\circ} \mathrm{C}$ to $200^{\circ} \mathrm{C}$.

It was hypothesized that the interface conductance increase with the bundle bulk average temperature could be due to the thermal expansion of conductors and their jackets within the bundle. As wire bundles are constrained on their exterior by an external jacket and/or tie wraps, an increase in the bundle bulk average temperature results in higher contact normal forces between adjacent wire jackets. It is reasoned that even local heating within a bundle will result in expansion of the affected conductors and jackets resulting in a global effect across the bundle since the bundle is externally constrained. As a demonstration of this mechanism, a thermal-stress model was formulated based on the following assumptions:

a. Copper and the ETFE jacket expand with temperature in accordance with published coefficient of thermal expansion (CTE) data;

b. The bundle exterior radius was constrained to simulate the effect of an outer jacket and/or tie wrap.

c. Average temperature in the bundle was assumed constant for the analysis.

To test the hypothesis, a 19-wire bundle thermal stress model was built using MSC Patran ${ }^{\circledR}$, imported into MSC Mentat $^{\circledR} 2015$ and solved using MSC Marc ${ }^{\circledR}$ 2015. CTE data for, both, ETFE and copper were obtained from [5] and [6], respectively. Normal contact force, assumed to be zero at $20^{\circ} \mathrm{C}$, was recovered from the model over the temperature range of $20^{\circ} \mathrm{C}$ to $200^{\circ} \mathrm{C}$. The calculated contact normal force for $200^{\circ} \mathrm{C}$ the case is presented in Figure 5. Both, linear and parabolic fits to the normal contact force versus bundle bulk average temperature curve yielded excellent fits with $R^{2}=$ 0.9951 and $R^{2}=0.9979$, respectively. As the analysis showed, as bundle bulk average temperature increases, so too does the normal contact force which supports the hypothesis that contact conductance between adjacent wire jackets may be due to increasing normal contact force induced by temperature rise in the constrained bundle.

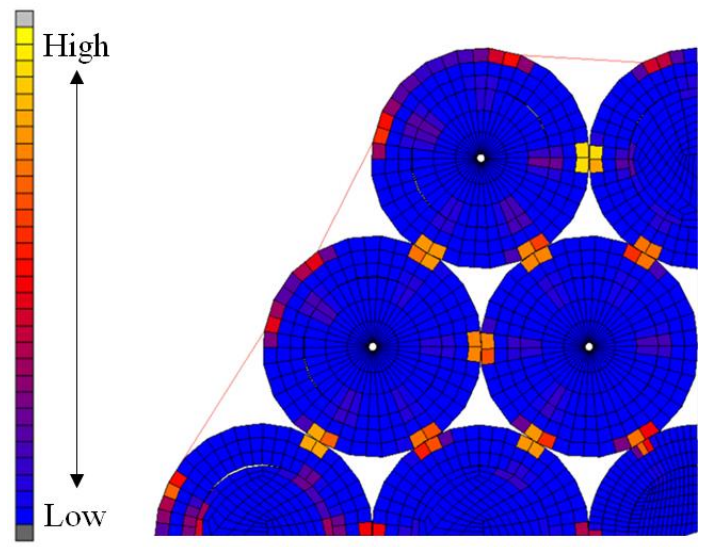

Fig. 5: Predicted normal contact force for a wire bundle bulk average temperature of $200^{\circ} \mathrm{C}$. 


\subsubsection{Air Conductance at Ambient Pressure}

Correlation of the wire bundle thermal models at ambient pressure required adjustment of the wire jacket-to-wire jacket interface conductance which was shown to be a function of bundle bulk average temperature in the previous section. In the presence of gravity, air will rise as it is warmed due to buoyancy driven forces. However, if the region in which the air is contained is small, there is insufficient space for convection to develop and the resulting heat transfer occurs via gas conduction. To determine whether air conduction or buoyancy driven convection is the driving heat transfer mode, the Rayleigh number, $R a$ is calculated by:

$$
R a=\frac{g \beta_{a i r} \Delta T_{a i r} L_{g a p}^{3}}{v_{a i r} \alpha_{a i r}}
$$

where $g$ is the acceleration due to gravity, $\beta_{\text {air }}$ is the air volume coefficient of expansion, $\Delta T$ is the temperature difference across the air gap, $L_{\text {gap }}$ is the characteristic dimension of the interstitial region between wires, $v_{\text {air }}$ is the kinematic viscosity for air, and $\alpha_{\text {air }}$ is the thermal diffusivity of air.

When $R a>\sim 1000-2000$, convection is possible. For a gap between three 22 AWG wires (shown in Figure 6) and an assumed air temperature of $80{ }^{\circ} \mathrm{C}$ and air properties from [7], $R a \ll 1$ and it is concluded that heat transfer is via air conduction.

A finite element approximation of the air gap formed by three adjacent wire jackets was used to estimate the effect of air conduction between wires. In the model, the leftmost boundary of the air region is heated while the other two boundaries were held at a constant boundary temperature of $80{ }^{\circ} \mathrm{C}$. Steady state analysis was performed and temperature distributions at the left hand boundary are shown on the right hand side of Figure 6.

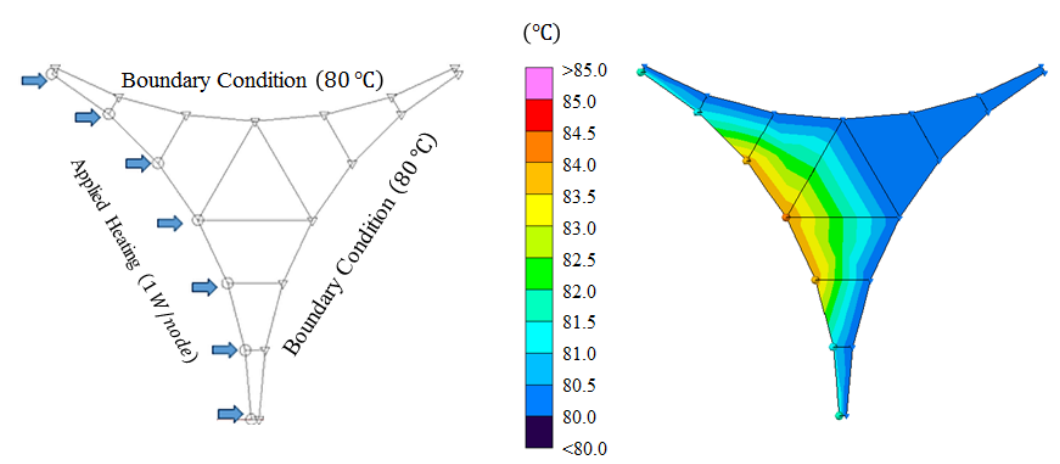

Fig. 6: Finite element model configuration for air conduction study.

As can be seen from the steady state analysis results, temperatures on the heated wire jacket range from $81.13{ }^{\circ} \mathrm{C}$ to $84.04{ }^{\circ} \mathrm{C}$. A representative average temperature is $82.5{ }^{\circ} \mathrm{C}$. For this air conduction analysis, it is noted that the heat transfer between the heated wire and the adjacent two wires is approximated by:

$$
\dot{Q}_{\text {air }} \approx G_{\text {air }, \text { total }} \Delta T \approx 2 G_{\text {air }} \Delta T
$$

For the case analyzed, $G_{\text {air }} \approx 1.4 \mathrm{~W} /{ }^{\circ} \mathrm{C}$.

When comparing aggregate interface conductance for the air and vacuum cases, the difference between the two values is representative of the heat transfer contribution of air conduction. At $80{ }^{\circ} \mathrm{C}$, the difference between the air and vacuum cases was determined to be on the order of $1 \mathrm{~W} /{ }^{\circ} \mathrm{C}$ which is in general agreement with the calculation.

\subsubsection{Aggregate Interface Conductance}

Analysis in the preceding sections showed that heat transfer between adjacent wire jackets within a bundle varies with, both, temperature and the presence of air. The effects were combined to form interpolation arrays used in the thermal model depending on whether an in-air or in-vacuum case is analyzed and is shown in Figure 7. The blue curve represents the array of data used for correlation of the in-air cases whereas the orange squares represent correlation values derived 
from the in-vacuum test data. Note that no curve was drawn between the orange data points as only two test points were available for correlation. However, it is expected that any curve connecting the two vacuum points will lie on or below the blue curve due to the absence of air conduction between wire jackets in the vacuum case. At high temperatures, the best correlation was found assuming the same vacuum conductance as the in-air case suggesting that at higher temperatures, contact conductance becomes the predominant heat transfer mechanism.

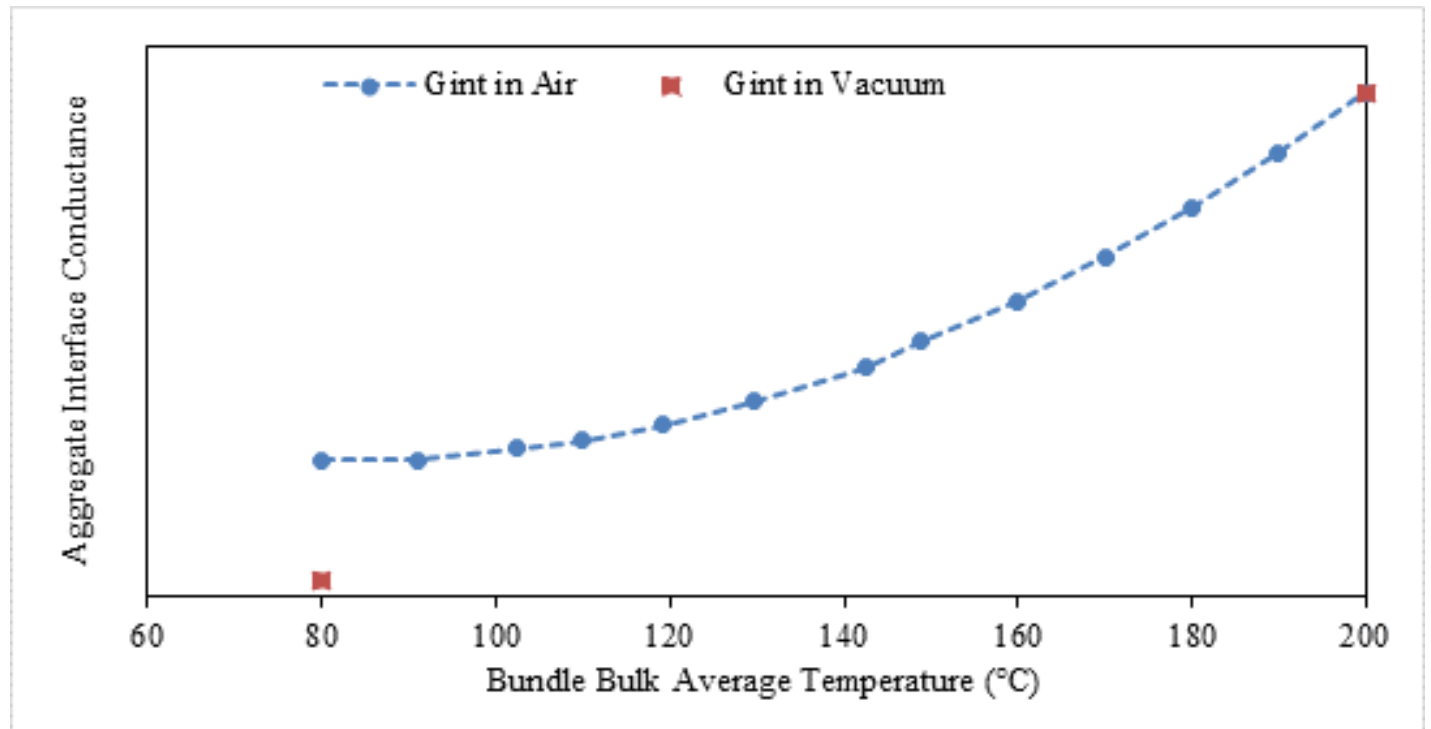

Fig. 7: Aggregate interface conductance for in-air and vacuum cases.

\subsection{Complex Wire Bundle Thermal Model Builder}

The Complex Wire Bundle Thermal Model Builder was developed due to the aforementioned limitations of the bundle spreadsheet model discussed in [4]. Specifically, due to Microsoft Excel® matrix inversion limitations, a maximum of 50 wire bundle elements could be modeled. Additionally, for vacuum analyses, the convergence of the temperature solution algorithm was not achieved for very low external bundle emittances.

The Complex Wire Bundle Thermal Model Builder also uses Microsoft Excel® but only as a "front end" allowing users to specify wire bundles comprised of up to 150 elements where an element may be a wire, a sub-bundle jacket or an outer jacket. When a user specifies a wire, the model building logic creates a wire conductor as well as an insulation jacket for each wire specified. Users may specify current values for each individual wire, wire gauge, jacket type, proximity to adjacent wires within the bundle, external environment (air or vacuum) and environment temperature. The tool uses Microsoft Excel ${ }^{\circledR}$ functions and Microsoft Visual Basic ${ }^{\circledR}$ routines to create an equivalent thermal network model in the Cullimore and Ring Technologies Systems Improved Numerical Differencing Analyzer (SINDA) format.

\subsection{Comparison of Wire Bundle Analytical Models with a Derating Standard}

In order to determine the feasibility of applying wire bundle thermal models as a tool for bundle derating, three wire bundle models were analyzed and compared with [1]. Wire bundles comprised of seven, nineteen and thirty-seven 22 AWG wires, depicted in Figure 8, were modeled using the Complex Wire Bundle Thermal Model Builder and SINDA input files were generated. Minor edits were made to the SINDA files to allow for user adjustment for bundle external jacket emittance, wire resistance per unit length and wire current.

The selected bundle sizes allowed comparison to the derating procedure over a reasonable range of bundle sizes. Additionally, the selected bundle sizes afforded a symmetry to aid the modeling process whereby each configuration had a single, centrally-located conductor which resulted in the highest predicted temperature. 


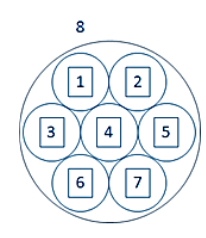

7-Wire

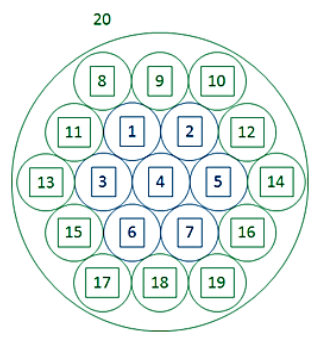

19-Wire

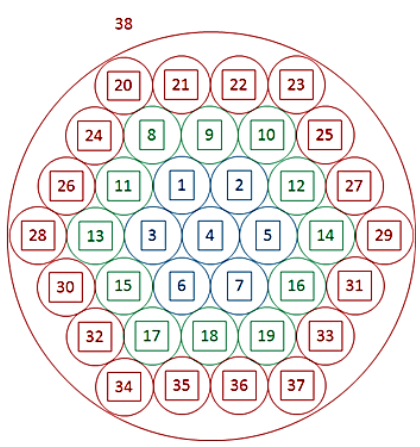

37-Wire

Fig. 8: Schematic of the 7-, 19- and 37-wire analysis configurations.

Steady state analysis was performed to determine the background current required in each wire that would result in a predicted central conductor temperature of $200^{\circ} \mathrm{C}$. The derating procedure in [1] was applied to determine ampacity for similarly sized bundles assuming a 100 percent loaded bundle in air at atmospheric pressure at $20{ }^{\circ} \mathrm{C}$. A comparison of the results is presented in Table 1.

Table 1: Comparison of model-predicted maximum allowable current with derating procedure.

\begin{tabular}{|c|c|c|c|}
\hline & \multicolumn{3}{|c|}{ Maximum Current per Wire to Attain $200{ }^{\circ} \mathrm{C}$ on Hottest Wire (A) } \\
\hline Configuration & $\begin{array}{c}\text { Model Prediction } \\
\text { Measured } R_{l}, \text { High } \varepsilon\end{array}$ & $\begin{array}{c}\text { Model Prediction } \\
\text { Specification } R_{l}, \text { High } \varepsilon\end{array}$ & $\begin{array}{c}\text { SAE 50881 F (Section 6.7) } \\
\text { [Ref. 8] }\end{array}$ \\
\hline 7 Wire & 10.26 & 10.00 & 9.68 \\
\hline 19 Wire & 7.54 & 7.18 & 6.34 \\
\hline 37 Wire & 6.12 & 5.90 & 4.71 \\
\hline
\end{tabular}

Two analysis cases were studied for each bundle size. The first case assumed the measured resistance per unit length $\left(R_{l}\right)$ whereas the second case assumed the maximum allowable $R_{l}$ per the wire specification (e.g., [8]). For both cases, a high emittance external bundle jacket $(\varepsilon=0.93)$ was assumed to demonstrate the difference between what is allowed per the specification and the potential gain by using a measured value. It is important to note that while the model builder has produced models that have been correlated to test data, the analysis discussed here has not been corroborated with test data and is only meant to serve as a comparison with a published derating standard. Future work will aim to obtain such data.

It should also be noted that different standards may rely on different assumptions. At the very least, some assumptions are not stated or are vague and would benefit from clarification. For instance, is unknown whether values resulting from the application of [1] include any temperature margin. This makes a direct comparison between the model predictions and the standard impossible until additional test data are obtained.

\section{Conclusion}

Improvements to existing wire bundle thermal models for ampacity determination have been presented. Components of wire jacket-to-wire jacket heat transfer consist of direct contact conductance, thermal radiation and, for in-air cases, air conduction. The heat transfer mechanisms have been implemented in a Complex Wire Bundle Thermal Model Builder. A large bundle model was correlated to test data resulting in data to correlate the wire jacket-to-wire jacket heat transfer. Subsequently, three bundles of various sizes were modeled and compared to a derating standard with encouraging results. Additional test data should be pursued and correlated to corresponding models to determine the potential for wider applicability of analysis as a tool for wire bundle derating. 


\section{Acknowledgements}

Acknowledgement is given for test support provided by Mr. Thad Johnson, Mr. Lawrence Ludwig, Mrs. Tamara Dodge, Mr. Dan Ciarlariello and Mr. Lawrence Batterson of the NASA - Kennedy Space Center.

\section{References}

[1] Aerospace Standard, Wiring Aerospace Vehicle, AS 50881 Revision F, SAE International, 2015.

[2] R. C. van Benthem, W. de Grave, F. Doctor, S. Taylor, K. Nuyten, and P. A. J. Dit Routier, "Thermal Analysis of Wiring Bundles for Weight Reduction and Improved Safety," AIAA 2011-5111, International Conference on Environmental Systems, Portland, OR, 2011.

[3] A. Ilgevičius, H. D. Liess, "Thermal Analysis of Electrical Wires by Finite Volume Method," ISSN 1392-1215 Elektronika ir Elektrotechnika, vol. 4, no. 46, pp. 87-92, 2003.

[4] S. L. Rickman and C. J. Iannello, "Heat Transfer Analysis in Wire Bundles for Aerospace Vehicles," in WIT Transactions on Engineering Sciences, Heat Transfer 2016, Wessex Institute, UK, vol. 106, pp. 53-63, 2016.
[5]
Material
Property
Data
for ETFE,
[Online].
Available: http://www.matweb.com/search/datasheet.aspx?matguid=9a8a721ac9f643389e0e793ea3abf5d3.

[6] MatWeb Material Property Data for Copper, Annealed, [Online]. Available: http://www.matweb.com/search/datasheet.aspx?matguid=9aebe83845c04c1db5126fada6f76f7e

[7] J. P. Holman, Heat Transfer, 5th Edition, New York: McGraw-Hill Book Company, p. 542, 1981.

[8] SAE-AS22759-32-33_6-21-13.pdf, [Online]. Available: http://www.rsccaerodefence.com 